\title{
Influence of the Primary Tumor Location on the Pattern of Synchronous Metastatic Spread in Patients with Stage IV Colorectal Carcinoma, According to the $8^{\text {th }}$ Edition of the AJCC Staging System
}

\author{
Sorin T. Alexandrescu ${ }^{1,2}$, Diana T. Anastase ${ }^{3}$, Razvan T. Grigorie ${ }^{1}$, Cristian A. Zlate ${ }^{1}$, Stefan Andrei ${ }^{2,4,5}$, Radu Costea $^{2}$, \\ Iulia M. Gramaticu ${ }^{6}$, Adina E. Croitoru ${ }^{6,7}$, Irinel Popescu ${ }^{1,7}$
}

\author{
1) Dan Setlacec Centre of \\ General Surgery and Liver \\ Transplantation, Fundeni \\ Clinical Institute, Bucharest, \\ Romania; \\ 2) Carol Davila University \\ of Medicine and Pharmacy, \\ Bucharest, Romania; \\ 3) Department of Plastic \\ and Reconstructive Surgery, \\ Emergency Clinical Hospital, \\ Bucharest, Romania; \\ 4) $1^{\text {st }}$ Department of \\ Anesthesia and Intensive Care, \\ Fundeni Clinical Institute, \\ Bucharest, Romania; \\ 5) Paris Sud XI University, \\ Faculty of Medicine, Paris, \\ France; \\ 6) Department of Oncology, \\ Fundeni Clinical Institute, \\ Bucharest, Romania; \\ 7) Faculty of Medicine, \\ Titu Maiorescu University, \\ Bucharest, Romania
}

\begin{abstract}
Address for correspondence:
Adina E. Croitoru, MD, PhD

Department of Oncology,

Fundeni Clinical Institute,

Soseaua Fundeni, 258, Sector

2, 022328, Bucharest, Romania

adinaecroitoru2019@gmail.com
\end{abstract}

Received: 15.06.2020

Accepted: 02.10.2020

\section{ABSTRACT}

Background \& Aims: The correlations between primary tumor location (right colon cancer - RCC, left colon cancer - LCC and rectal cancer - RC) and the incidence of metastatic sites are scarce and divergent. The current study is the first which compares the pattern of metastatic distribution (M1a: metastasis to one organ/site, excluding peritoneum; M1b: two or more metastatic sites; M1c: peritoneal metastases) between RCC, LCC and RC, respectively.

Methods: All patients operated for colorectal cancer (CRC) between January 2006 and December 2015 were analyzed to assess the primary tumor location, the presence and site of synchronous metastases. Univariate analysis determined the statistical significance of association between each CRC location and the metastatic pattern. Multinomial logistical regression model compared the prevalence of each metastatic pattern for each CRC location.

Results: Out of 5,107 patients, 1,318 (25.80\%) had metastases on the moment of CRC diagnosis. There were no statistically significant association between the metastatic pattern and the patients' gender $(\mathrm{M} 1 \mathrm{a}, \mathrm{p}=0.321 ; \mathrm{M} 1 \mathrm{~b}$, $\mathrm{p}=0.539 ; \mathrm{M} 1 \mathrm{c}, \mathrm{p}=0.417$, Chi-square) or patients' age ( $\mathrm{p}=0.616 \mathrm{Mann}$-Whitney U-test). RC had a significant higher relative risk for $\mathrm{M} 1 \mathrm{a}(\mathrm{RR}$ of $1.437, \mathrm{p}=0.014)$ and a lower relative risk for $\mathrm{M} 1 \mathrm{c}(\mathrm{RR}$ of $0.564, \mathrm{p}=0.001)$, compared to LCC. On the contrary, compared with LCC, the RCC showed a significant lower relative risk for $\mathrm{M} 1 \mathrm{a}$ (RR of $0.673, \mathrm{p}=0.006)$ and a higher relative risk for M1c (RR of 1.834, $\mathrm{p}=0.0001)$.

Conclusion. There is a strong correlation between the primary location of CRC and the pattern of the metastatic spread, with potential prognostic implications.

Key words: colorectal cancer - rectal cancer - location - metastases - AJCC 8th Edition Cancer Staging incidence.

Abbreviations: AJCC: American Joint Committee on Cancer; BRAF: v-raf murine sarcoma viral oncogene homolog B; CRC: colorectal cancer; CT: computed tomography; DW-MRI: diffusion-weighted - magnetic resonance imaging; IQR: interquartile range; LCC: left colon cancer; M1a: metastasis to one organ/site, excluding peritoneum; M1b: two or more metastatic sites without peritoneal involvement ; M1c: peritoneal metastases; mCRC: metastatic CRC; OS: overall survival; PET: positron emission tomography; RCC: right colon cancer; RC: rectal cancer; RR: relative risk; SD: standard deviation.

\section{INTRODUCTION}

Colorectal cancer (CRC)

is an important issue in public health, being the most frequent oncological pathology of the gastrointestinal tract. Until the last years, CRC was considered a unitary entity, but quite recently, some studies have demonstrated that there are differences in the prognosis of patients with metastatic colorectal cancer (mCRC), depending on the location of the primary tumor (between the right colon vs. the left colon and the rectum) [1-3]. During recent years, some explanations have emerged, such as the different embryological origin of the right colon versus the left colon and the rectum, the distinct pattern of somatic mutations in adenocarcinomas located on the right side versus left-sided CRC and the differences in the microbiota [1-3]. However, these explanations revealed some dissimilarities between the right colon tumors, on one hand, and the left colon and rectal tumors (altogether), on the other hand. Thus, little is known regarding the differences in prognostic and biologic 
behavior between right colon cancer (RCC), left colon cancer (LCC) and rectal cancers (RC), as distinct subsets of colorectal malignancies.

Moreover, the 8th Edition of American Joint Committee on Cancer (AJCC), Cancer Staging Manual, introduced the M1c category (stage IVC CRC) to specifically define the peritoneal metastases, because these patients portend a poorer prognosis than those with CRC and metastases in site(s) other than the peritoneum [4-8]. Thus, in present, stage IV CRC is divided into three substages: stage IVA (M1a category: metastases involving one organ/site, except for peritoneum); stage IVB (M1b category: two or more metastatic sites, without peritoneal involvement); stage IVC (M1c category: peritoneal metastases, with or without other metastatic sites). The higher frequency of peritoneal metastases in patients with colon cancer vs. rectal carcinoma was recently showed by Holch et al. [9]. However, the pattern of metastatic spread (M1a, M1b, M1c) in patients with stage IV CRC, depending on the location of the primary tumor has not been assessed, so far.

The aim of this study was to evaluate the incidence of metastatic disease in newly diagnosed patients with CRC and to perform a correlation between the primary tumor location and the distribution of the three categories of metastatic diseases (M1a, M1b, and M1c). To the best of our knowledge, this is the first study which has evaluated the correlation between the pattern of metastatic spread in patients with stage IV CRC (according to the 8th edition of AJCC staging system) and the primary tumor location (right colon, vs. left colon, vs. rectum).

\section{METHODS}

The data of all the patients operated for CRC in Fundeni Clinical Institute, between January 2006 and December 2015 were retrospectively analyzed. The clinical and pathological data were acquired from the operation database of the center, from the clinical observation charts of patients, and from the pathology examination registry. The patients presenting other types of malignancies rather than carcinomas were excluded from the study. Also, the patients with squamous cell carcinomas of the anal canal were excluded.

The location of the primary tumor, the presence or absence of synchronous metastases and, if metastases were present and their locations were recorded for each patient with CRC. The metastases were considered as synchronous with the CRC if they were diagnosed before or during the operation performed for the primary tumor.

It is worth mentioning that in our institution even patients with unresectable mCRC underwent surgery, either with the aim of primary tumor resection or with the goal of performing a stoma or by-pass for the primary tumor's complications. The decision to remove the primary tumor in patients with unresectable mCRC and uncomplicated primary tumors is based on the evidence (level B and C) which revealed that primary tumor resection improved overall survival in such patients $[10,11]$.

The performance of this retrospective study was approved by the Ethics Committee of Fundeni Clinical Institute, Bucharest, under the number 30767/11.06.2020.
The diagnosis of CRC was established by preoperative colonoscopy in most patients. In a small number of patients, the primary tumor was diagnosed during laparotomy performed for complications of CRC (bowel obstruction, hemorrhage, perforation). The diagnostic work-up for the detection of synchronous metastases in patients newly diagnosed with CRC consisted of a computed tomography (CT) scan of the thorax, abdomen and pelvis. If the results of this investigation were uncertain, magnetic resonance imaging (MRI) or positron emission tomography (PET)-CT was recommended. In most patients with complications of the primary tumor, operated on an emergency basis, if the CT scan was not performed before the operation, the investigation was done postoperatively (before discharging the patient). During the surgery, a complete exploration of the abdominal cavity (including parietal peritoneum, small bowel, colon, liver and spleen) was performed, even when preoperative CT scan did not reveal synchronous metastases. The exploration of the abdominal cavity has been performed either by laparoscopy, or by laparotomy. Laparotomy was performed through a median incision, even in patients with preoperatively known obstructive primary tumor and unresectable synchronous metastases. Also, in such patients, the complete exploration of the peritoneal cavity is mandatory, because sometimes the cause of bowel obstruction could be represented by peritoneal metastases involving the small bowel, and in such instances the colostomy would be futile. Both laparoscopy and median laparotomy allow for a complete visual evaluation of the peritoneal surface. Whenever a peritoneal/ovarian lesion raised the suspicion of tumor seeding, a biopsy was performed with pathological examination to confirm the metastatic disease. Intraoperative exploration also involved visual and palpatory/hepatic evaluation of the liver surface. If suspicions regarding the presence of liver metastases were raised, intraoperative ultrasound was performed. When liver lesions were still equivocal at intraoperative ultrasound, a biopsy was performed to confirm or rule out hepatic metastases. Central nervous system metastases were diagnosed by preoperative CT scan or MRI, performed when suggestive symptoms/ signs were present. The diagnosis of bone metastases was established by either CT scan/MRI or bone scintigraphy. Scintigraphy has been done only in patients with suggestive symptoms or in patients whose CT scan/MRI raised this suspicion.

Although most patients were diagnosed with CRC before the operation, the location of the primary tumor was established based on the intraoperative findings. Thus, the tumors located in the cecum, ascending colon and transverse colon were considered as RCC, while the LCC included the tumors located at the splenic flexure, descending colon and sigmoid. Rectal cancer was located in the large intestine below the peritoneal reflection.

The subclassification of patients with mCRC was performed according to the AJCC $8^{\text {th }}$ edition for TNM staging of colorectal carcinoma [12]. Thus, the patients with metastasis confined to one organ or site, without peritoneal metastasis were included in the M1a group. The M1b group included the patients with metastases to two or more sites or organs, but without peritoneal metastasis. The presence of peritoneal 
metastases (alone or associated with other metastatic sites) led to the inclusion in the M1c group.

The data were recorded using Microsoft Excel ${ }^{\circledast}$ and the statistical analysis was performed using SPSS ${ }^{\circledast}$ (IBM SPSS Statistics for Windows, Version 21.0. Armonk, NY, IBM Corp). The incidence of metastatic disease was calculated for the entire cohort of patients, as well as for individual locations of the primary tumor (RCC, LCC and RC, respectively). Descriptive analyses were presented as mean \pm standard deviation (SD) or as median with [25\%-75\%] interquartile range (IQR), as appropriate. Normal distribution for continuous variables was evaluated by the Shapiro-Wilk test. The Mann-Whitney U-test was used for comparisons of non-parametric continuous variables. The Chi-square test was used to compare categorical variables. By univariate analysis, we first determined the statistical significance of the association between each type of CRC and the pattern of metastatic disease. Secondly, we used a multinomial logistical regression model to compare the prevalence of each type of CRC in each Metastatic substage. A p value less than 0.05 was considered statistically significant.

\section{RESULTS}

Between January 2006 and December 2015, 5,213 patients with CRC underwent surgery in a tertiary center. Out of these patients, 106 presented synchronous primary tumors, located either in the right colon and left colon, or in the colon and rectum and were excluded from the analysis. Thus, the incidence of synchronous metastases was assessed on 5,107 patients with only one primary tumor: 1,213 patients with RCC, 1,963 with LCC and 1,931 with RC (Fig. 1).

The highest incidence of stage IV disease was observed in patients with LCC $(31.4 \%, 617 / 1963)$ and the lowest incidence in RC patients $(20.1 \%, 387 / 1931)$. Out of 1,213 patients with RCC, 314 had stage IV disease (25.9\%). The locations of synchronous metastases, as well as the incidences of specific metastatic sites according to the primary tumor location, are presented in Table I. Incidences of liver metastases and those of peritoneal metastases were significantly higher in colon cancers than in rectal carcinomas $(18.2 \%$ and $8.18 \%$ vs. $13.9 \%$ and $3 \%$, respectively; $\mathrm{p}=0.0001$ ). On the contrary, RCs were associated with a significantly higher incidence of lung

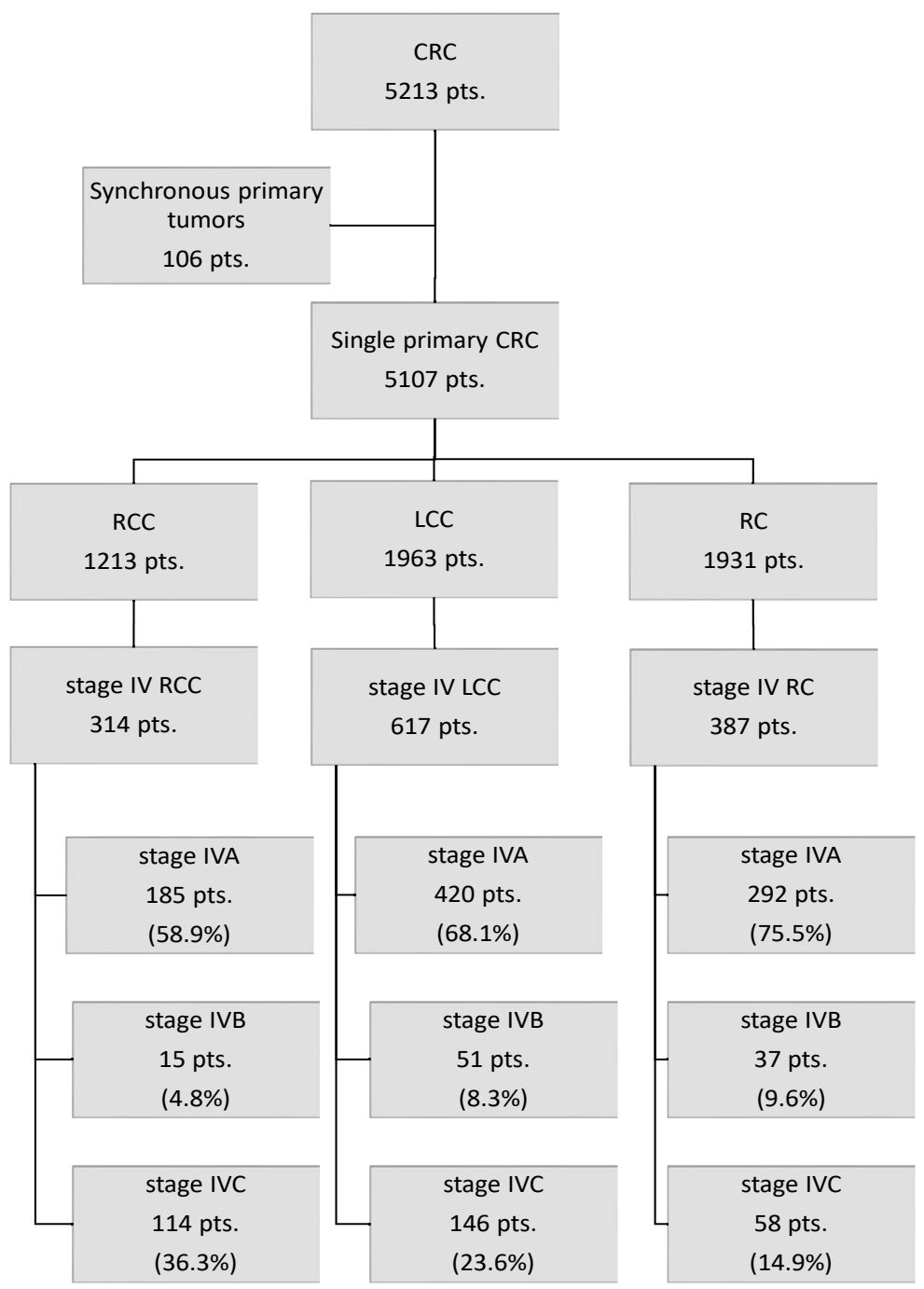

Fig. 1. Flow-chart of patients operated for colorectal cancer. Frequencies of metastatic pattern, according to the $8^{\text {th }}$ edition of the AJCC staging system (M1a/stage IVA, M1b/stage IVB, M1c/stage IVC). RCC: right colon cancer, LCC: left colon cancer, RC: rectal cancer. 
Table I. Incidence of each type of synchronous metastases, according to the primary tumor location. Comparison of incidences for each metastatic site between the right colon cancer vs. left colon cancer, and colon cancers vs. rectal carcinomas, respectively.

\begin{tabular}{|c|c|c|c|c|c|c|}
\hline & $\begin{array}{c}\text { Right colon } \\
\text { cancer } \\
\mathrm{n}=1213\end{array}$ & $\begin{array}{c}\text { Left colon } \\
\text { cancer } \\
\mathrm{n}=1963\end{array}$ & $\begin{array}{c}\mathrm{p} \\
\text { (Right colon } \\
\text { vs. Left colon) }\end{array}$ & $\begin{array}{c}\text { Colon cancer } \\
\mathrm{n}=3,176\end{array}$ & $\begin{array}{c}\text { Rectal cancer } \\
\mathrm{n}=1,931\end{array}$ & $\begin{array}{c}\mathrm{p} \\
\text { (Colon vs. } \\
\text { Rectum) }\end{array}$ \\
\hline No-metastases & $899(74.1 \%)$ & $1346(68.6 \%)$ & $0.0009^{*}$ & $2245(70.7 \%)$ & $1544(79.9 \%)$ & $0.0001^{*}$ \\
\hline Metastases & $314(25.9 \%)$ & $617(31.4 \%)$ & & $931(29.3 \%)$ & $387(20.1 \%)$ & \\
\hline M1a & $185(15.25 \%)$ & $420(21.39 \%)$ & $0.0001^{*}$ & $605(19 \%)$ & $292(15.1 \%)$ & $0.0004^{*}$ \\
\hline Liver & $172(14.17 \%)$ & $407(20.73 \%)$ & $0.0001^{*}$ & $579(18.2 \%)$ & $269(13.9 \%)$ & $0.0001^{*}$ \\
\hline Lung & $4(0.33 \%)$ & $5(0.25 \%)$ & 0.7388 & $9(0.28 \%)$ & $14(0.72 \%)$ & $0.0297^{*}$ \\
\hline Lymph nodes & $6(0.49 \%)$ & $6(0.3 \%)$ & 0.3918 & $12(0.37 \%)$ & $5(0.25 \%)$ & 0.6189 \\
\hline Other sites & $3(0.24 \%)$ & $2(0.1 \%)$ & 0.3766 & $5(0.15 \%)$ & $4(0.2 \%)$ & 0.7371 \\
\hline M1b & $15(1.23 \%)$ & $51(2.6 \%)$ & $0.0099^{*}$ & $66(2.07 \%)$ & $37(1.91 \%)$ & 0.7584 \\
\hline Liver + Lung & $9(0.74 \%)$ & $30(1.52 \%)$ & 0.0667 & $39(1.22 \%)$ & $27(1.39 \%)$ & 0.6110 \\
\hline Liver + Lymph nodes & $2(0.16 \%)$ & $9(0.45 \%)$ & 0.2228 & $11(0.34 \%)$ & $3(0.15 \%)$ & 0.2743 \\
\hline Liver + Other sites & $4(0.33 \%)$ & $8(0.4 \%)$ & 1 & $12(0.37 \%)$ & $6(0.31 \%)$ & 0.8105 \\
\hline Other sites & $0(0 \%)$ & $4(0.2 \%)$ & 0.3046 & $4(0.12 \%)$ & $1(0.05 \%)$ & 0.6560 \\
\hline M1c & $114(9.4 \%)$ & $146(7.43 \%)$ & 0.0534 & $260(8.18 \%)$ & $58(3 \%)$ & $0.0001^{*}$ \\
\hline Peritoneal only & $45(3.7 \%)$ & $65(3.31 \%)$ & 0.5505 & $110(3.46 \%)$ & $19(0.98 \%)$ & $0.0001^{*}$ \\
\hline Peritoneal + Other Sites & $69(5.7 \%)$ & $81(4.12 \%)$ & $0.0477^{*}$ & $150(4.72 \%)$ & $39(2.01 \%)$ & $0.0001^{*}$ \\
\hline
\end{tabular}

M1a: metastasis to one organ/site, excluding peritoneum; M1b: two or more metastatic sites without peritoneal involvement; M1c: peritoneal metastases; ${ }^{\star}: \mathrm{p}<0.05$.

metastases, than colon tumors $(0.72 \%$ vs. $0.28 \%$, respectively; $\mathrm{p}=0.0297)$.

In LCC the incidence of liver metastases was significantly higher than in RCC (20.7\% vs. $14.1 \%, p=0.0001)$, while the incidence of peritoneal metastases combined with other metastatic sites was significantly lower in LCC than in RCC ( $4.1 \%$ vs. $5.7 \%$, respectively; $\mathrm{p}=0.0477$ ) (Table I).

From this cohort of 5,107 patients, 1,318 patients $(25.80 \%)$ had metastases on the moment of CRC diagnosis (stage IV disease) and were included in the statistical analysis (Fig. 1). The pattern of metastatic distribution (according to the 8th edition of AJCC staging system) was evaluated among the 1,318 patients with stage IV CRC. In this group, 799 (60.6\%) patients were males and $519(39.4 \%)$ were females and the median age was 62 years, IQR: 55-70 years. Out of 1,318 the patients with mCRC, 897 (68.1\%) patients presented with M1a substage, 103 (7.8\%) with M1b, and 318 (24.1\%) with M1c, respectively.

Univariate analysis was performed to evaluate the association between age, gender and primary tumor location with each metastatic substage, among the metastatic patients. The results are resumed in Table II. There were no statistically significant associations between metastatic substages and patients' gender (M1a, $\mathrm{p}=0.321 ; \mathrm{M} 1 \mathrm{~b}, \mathrm{p}=0.539 ; \mathrm{M} 1 \mathrm{c}, \mathrm{p}=0.417$; Chi-square), or patients' age ( $\mathrm{p}=0.616$; Mann-Whitney U-test).

The multinomial regression analysis for each metastatic substage is presented in Table III. Compared with LCC, the RCC showed a higher RR for M1c pattern (RR: 1.834, 95\%CI: 1.362-2.471, $\mathrm{p}<0.001$ ) and a lower RR for M1a pattern (RR:0.673, 95\%CI: 0.507-0.894, p=0.006). Furthermore, compared with LCC, the RC had a higher statistically significant RR for M1a pattern (RR: 1.437,95\%CI: 1.077-1.918, p=0.014), and a lower RR for M1c pattern (RR: 0.564, 95\%CI: 0.402-0.791, $\mathrm{p}=0.001)$. Neither RCC $(\mathrm{p}=0.057)$, nor $\mathrm{RC}(\mathrm{p}=0.425)$ had a statistically significant relative risk for developing M1b substage, compared to LCC (Fig. 2).
Table II. Univariate analysis of the relation between each type of colorectal cancer and each metastatic disease substage (M1a, M1b, M1c).

\begin{tabular}{lcccccc}
\hline $\begin{array}{l}\text { Type of } \\
\text { cancer }\end{array}$ & $\begin{array}{c}\text { M1a } \\
(\mathrm{n})\end{array}$ & $\begin{array}{c}\mathrm{p}(\mathrm{Chi}- \\
\text { square })\end{array}$ & $\begin{array}{c}\text { M1b } \\
(\mathrm{n})\end{array}$ & $\begin{array}{c}\mathrm{p}(\mathrm{Chi}- \\
\text { square })\end{array}$ & $\begin{array}{c}\text { M1c } \\
(\mathrm{n})\end{array}$ & $\begin{array}{c}\mathrm{p}(\text { Chi- } \\
\text { square })\end{array}$ \\
\hline RCC & 185 & $0.0001^{*}$ & 15 & $0.022^{*}$ & 114 & $0.0001^{*}$ \\
LCC & 420 & 0.992 & 51 & 0.567 & 146 & 0.712 \\
RC & 292 & $0.0001^{*}$ & 37 & 0.128 & 58 & $0.0001^{*}$ \\
\hline
\end{tabular}

RCC: right colon cancer; LCC: left colon cancer; RC: rectal cancer; n: number of patients; ${ }^{\star}: \mathrm{p}<0.05$.

\section{DISCUSSION}

Although some evidence suggested that in stage IV CRC certain metastatic sites were more frequently associated with the primary tumor location [13-15], other metastatic sites did not regularly correlate with the specific location of CRC. The results of the present study are in line with those of other reports, revealing that patients with stage IV rectal primaries are more likely to present with lung metastases than patients with stage IV colon cancers $[13,15]$. Regarding the incidence of isolated peritoneal or liver metastases, few authors observed that such locations of metastatic disease were more frequently associated with colon cancers than rectal carcinomas $[4,9,13$, 14], a similar result being reported by the current study. On the contrary, other associations between the primary tumor location and different combinations of metastatic sites were not uniformly reported. Moreover, such combinations of metastatic sites are difficult to be compared among different studies, due to the lack of standardization. Such a fragmented landscape did not allow a correlation between the primary tumor location and the prognosis of patients with mCRC.

To achieve a better standardization of the relationship between the primary tumor site and the pattern of metastases 
Table III. Multinomial regression results with consecutive M1 patterns as a dependent variable.

\begin{tabular}{|c|c|c|c|c|c|}
\hline \multicolumn{2}{|c|}{ Variables } & \multirow[t]{2}{*}{ Relative risk } & \multicolumn{2}{|c|}{$\begin{array}{l}\text { 95\% Confidence Interval for } \\
\operatorname{Exp}(B)\end{array}$} & \multirow[t]{2}{*}{$\mathrm{p}$} \\
\hline & & & Lower Bound & Upper Bound & \\
\hline \multirow[t]{7}{*}{ M1a } & Intercept & & & & 0.002 \\
\hline & Age & 0.995 & 0.984 & 1.006 & 0.370 \\
\hline & Right colon cancer & 0.673 & 0.507 & 0.894 & $0.006^{*}$ \\
\hline & Rectal cancer & 1.437 & 1.077 & 1.918 & $0.014^{*}$ \\
\hline & Left colon cancer (reference) & 1 & . & . & . \\
\hline & Female & 0.870 & 0.684 & 1.108 & 0.260 \\
\hline & Male (reference) & 1 & . & . & . \\
\hline \multirow[t]{7}{*}{ M1b } & Intercept & & & & 0.000 \\
\hline & Age & 1.003 & 0.985 & 1.022 & 0.751 \\
\hline & Right colon cancer & 0.562 & 0.311 & 1.018 & 0.057 \\
\hline & Rectal cancer & 1.199 & 0.768 & 1.873 & 0.425 \\
\hline & Left colon cancer (reference) & 1 & . & . & \\
\hline & Female & 1.205 & 0.796 & 1.823 & 0.378 \\
\hline & Male (reference) & 1 & . & . & $\cdot$ \\
\hline \multirow[t]{7}{*}{ M1c } & Intercept & & & & 0.000 \\
\hline & Age & 1.005 & 0.993 & 1.017 & 0.430 \\
\hline & Right colon cancer & 1.834 & 1.362 & 2.471 & $0.0001^{*}$ \\
\hline & Rectal cancer & 0.564 & 0.402 & 0.791 & $0.001^{*}$ \\
\hline & Left colon cancer (reference) & 1 & . & . & . \\
\hline & Female & 1.096 & 0.841 & 1.427 & 0.499 \\
\hline & Male (reference) & 1 & . & . & . \\
\hline
\end{tabular}

For abbreviations see table II.

distribution, the current study assessed the association between each of the three subclasses of stage IV CRC and the location of the primary tumor. Regarding the primary tumor location, apart from the differences in metastases distribution between the colon and rectum tumors, different biological behavior was observed between right-sided vs. left-sided CRC [16-18]. Thus, gradual change in mucosal immunogenicity, as well as the observation that the frequencies of CIMP+/MSI+/BRAF+

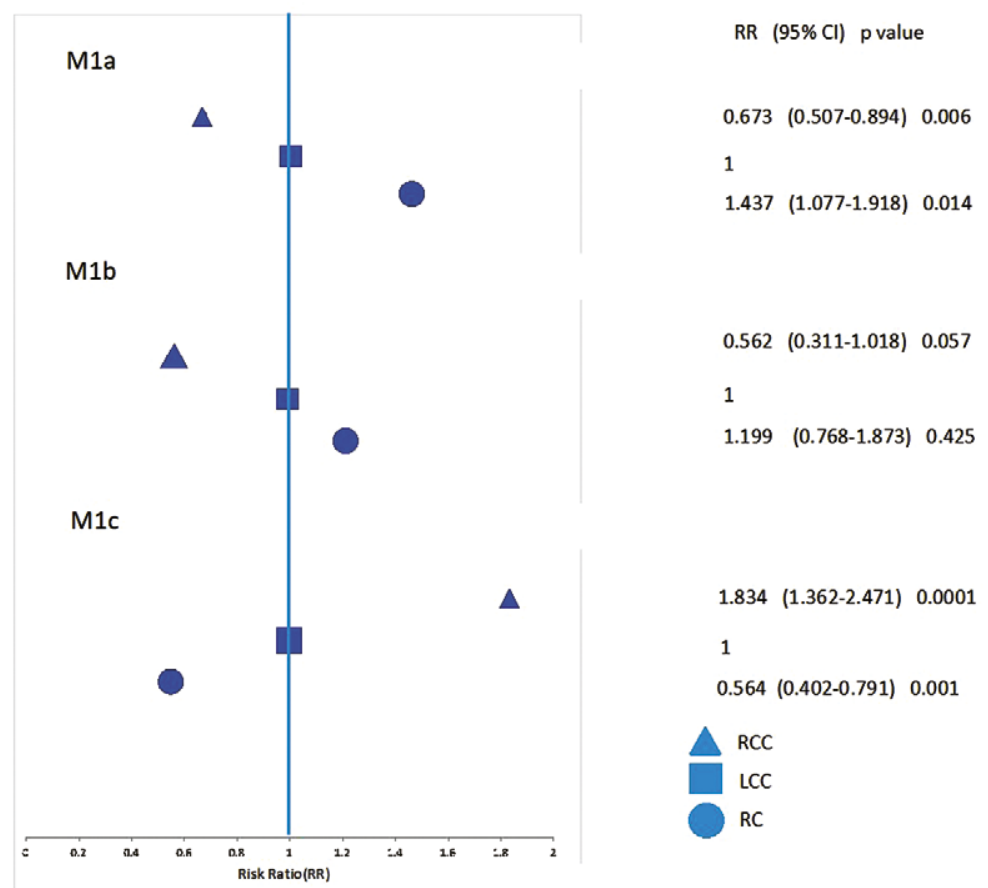

Fig. 2. Relative risk for development of each substage of metastatic disease (M1a, M1b, M1c), according to the primary tumor location. 
tumors gradually decreased from ascending colon to the rectum, without an abrupt change at splenic flexure [19], also suggested a different biological behavior between proximal colon tumors vs. distal colon cancers vs. rectal carcinomas [20]. For these reasons, in this study, the location of the primary tumor was divided into three categories.

Regarding the correlation between the primary tumor location and the metastatic pattern, RC had a significantly higher RR of developing M1a metastases than LCC and the later was associated with a significantly higher risk of M1a than RCC. RCC was associated with the highest RR of developing M1C (significantly higher than those of patients with LCC), while patients with RC had the lowest risk of M1c (significantly lower than LCC patients). Therefore, to the best of our knowledge, this is the first study which revealed a specific correlation between the primary tumor location and metastases distribution (according to the 8th edition of AJCC staging system).

A recent large study (which analyzed 10,553 patients with mCRC who underwent oncologic treatment in 14 phase 3 randomized trials) reported that $13 \%$ patients had peritoneal metastases [4], representing nearly half of the frequency of peritoneal involvement observed in the current study (24.1\%). Such an important discrepancy in the reported frequency of peritoneal metastases among different studies has already been observed, clinical trials under-representing peritoneal metastases (7.9-16.6\%) [21-25] compared to the autopsy or surgical series $(20.1-24 \%)$ [7, 26, 27]. The disproportionately lower frequency of peritoneal metastases in clinical trials than in surgical/autopsy series has two major explanations: systematic exclusion of such patients from clinical trials due to the difficult modality to assess the response to the oncologic therapy [8] and the challenging detection of peritoneal metastases in the absence of direct surgical observation [4]. Due to these drawbacks, the generalizability of the therapeutic results observed in randomized clinical trials (RCT) is reduced in patients with synchronous peritoneal metastases from CRC. To overcome this disadvantage, future RCTs should include more patients with peritoneal metastases, and routinely use newer imaging modalities [such as diffusion-weighted (DW)MRI and PET/CT) before treatment allocation and inclusion in clinical trials $[8,13,28]$. Because the relative risk for developing peritoneal metastases was significantly higher in RCC, in such patients it might be useful to perform either PET/CT or DWMRI before a therapeutic decision. Conversely, because RC was more frequently associated with stage IVA and presented less frequently peritoneal metastases, in such patients PET/CT might not bring additional information that would change the therapeutic strategy.

The associations between the primary tumor site and metastatic distribution might be a consequence of distinct pathologic and molecular features observed between RCC, LCC and RC. Thus, RCC tended to have more mucinous type carcinomas (7.4\% vs. 3.5\%; $\mathrm{p}=0.002)$ [29] and signet ring carcinomas than the left-sided tumors [30]. It was also observed that mucinous and signet ring carcinomas were significantly more frequently associated with peritoneal metastases, than other colorectal adenocarcinomas $(p<0.005)[1,26,29]$. These observations may explain the increased likelihood of M1c in patients with RCC compared to their counterparts with
LCC or RC. Beyond these histologic observations, a different genetic profile of the right-sided vs. left-sided vs. rectal tumors has already been reported and might explain the association between primary tumor location and the pattern of metastatic disease. The mutations in BRAF have been observed more frequently in RCC than in left-sided tumors (8.4\% vs. $2.9 \%$; $\mathrm{p}<0.001)[18,19,29]$ and a significantly higher proportion of patients with peritoneal metastasis had BRAF mutations compared to patients with non-peritoneal metastases $(\mathrm{p}=0.028)$ $[4,8]$. Thus, BRAF mutations seem to play a central role in the association between RCC and stage IVC.

The prevalent association, observed in the current study, between primary tumor location and the three subcategories of stage IV mCRC could also represent an explanation for the higher survival observed in left-sided mCRC than in rightsided tumors [1-3]. Thus, patients with metastatic RCC have the highest probability of developing stage IVC disease and the lowest probability for stage IVA disease. Patients with metastatic RC have the lowest risk of developing stage IVC disease, and the highest chance to present with stage IVA. Because overall survival (OS) rates of patients with mCRC decrease from stage IVA to stage IVB and further to stage IVC [4-8], the present study suggests that metastatic RC predicts a better life-expectancy compared to patients with metastatic LCC, who might have a better prognosis than RCC patients. Few recent studies reflected such a survival trend. A Korean group revealed that in stage IV CRC, the median OS of patients with right-sided colon tumors (13.7 months) was significantly shorter than the survival of patients whose tumors were located in the left colon (18.0 months) or rectum (19.9 months), respectively ( $\mathrm{p}=0.003$ ) [3]. Furthermore, two studies reported that metastatic $\mathrm{RC}$ had significantly higher OS rates compared to RCC $(\mathrm{p}<0.05)[1,2]$.

The current study has some inherent limitations, mainly because it was a retrospective analysis of a cohort of patients. As the study included only patients operated for CRC in a tertiary center, selection bias was possible. Thus, this study could have missed some patients with advanced metastatic disease who were not referred to surgery by the oncologists, especially if ECOG status was poor. However, the magnitude of this selection bias would have been negligible because the policy of the center was to perform resection of the primary tumor even in patients with preoperatively known unresectable metastases. This strategy was based on the observation that primary tumor resection improves OS even in asymptomatic patients whose metastases were not resected $[10,11]$. The incidence of synchronous metastases reported by the current study $(25.8 \%)$ was higher than those observed in autopsy or registry studies (16.3-21.3\%) $[7,14,26,27]$ suggesting that the proportion of patients with mCRC that were missed was insignificant. Furthermore, given the policy to operate most patients with CRC, this study offers the opportunity for a more accurate evaluation of the initial peritoneal involvement, which is under-scored in those studies including non-operated metastatic patients.

\section{CONCLUSIONS}

This is the first study which revealed the strong correlation between primary tumor location (right colon, left colon or 
rectum) and the three subclasses of metastatic CRC (M1a, $\mathrm{M} 1 \mathrm{~b}$ and M1c). Thus, RCC has the highest RR for M1c and the lowest RR for M1a, while RC has the highest RR for M1a and the lowest RR for M1c (the difference was significant between each two of the three locations: RCC vs. LCC vs. RC). This specific association between the primary tumor location and the three M1 subclasses could explain the poorer survival of patients with metastatic RCC compared to RC and even LCC.

Conflicts of interest: None to declare.

Authors' contribution: S.T.A. conceived and designed the study drafted the manuscript. D.T.A., R.T.G, C.A.Z. and I.M.G. collected the data. S.A., R.C. performed the statistical analysis. A.E.C. analyzed the data and drafted the manuscript. I.P. conceived and designed the study. All authors critically revised the manuscript, approved the final version to be published, and agreed to be accountable for all aspects of the work.

\section{REFERENCES}

1. Riihimäki M, Hemminki A, Sundquist J, Hemminki K. Patterns of metastasis in colon and rectal cancer. Sci Rep 2016;6:29765. doi:10.1038/ srep29765

2. Suthananthan AE, Bhandari M, Platell C. Influence of primary site on metastatic distribution and survival in stage IV colorectal cancer: Survival in metastatic colorectal cancer. ANZ J Surg 2018;88:445-449. doi:10.1111/ans.13969

3. Byun JH, Ahn JB, Kim SY, et al. The impact of primary tumor location in patients with metastatic colorectal cancer: a Korean Cancer Study Group CO12-04 study. Korean J Intern Med 2019;34:165-177. doi:10.3904/ kjim. 2016.348

4. Franko J, Shi Q, Meyers JP, et al. Prognosis of patients with peritoneal metastatic colorectal cancer given systemic therapy: an analysis of individual patient data from prospective randomised trials from the Analysis and Research in Cancers of the Digestive System (ARCAD) database. Lancet Oncol 2016;17:1709-1719. doi:10.1016/S14702045(16)30500-9

5. Franko J, Shi Q, Goldman CD, et al. Treatment of Colorectal Peritoneal Carcinomatosis With Systemic Chemotherapy: A Pooled Analysis of North Central Cancer Treatment Group Phase III Trials N9741 and N9841. J Clin Oncol 2012;30:263-267. doi:10.1200/JCO.2011.37.1039

6. Merkel S, Weber K, Croner RS, et al. Distant metastases in colorectal carcinoma: A proposal for a new M1 subclassification. Eur J Surg Oncol 2016;42:1337-1342. doi:10.1016/j.ejso.2016.03.034

7. Lemmens VE, Klaver YL, Verwaal VJ, Rutten HJ, Coebergh JW, de Hingh IH. Predictors and survival of synchronous peritoneal carcinomatosis of colorectal origin: A population-based study. Int J Cancer 2011;128:2717-2725. doi:10.1002/ijc.25596

8. Tseng J, Bryan DS, Poli E, Sharma M, Polite BN, Turaga KK. Underrepresentation of peritoneal metastases in published clinical trials of metastatic colorectal cancer. Lancet Oncol 2017;18:711-712. doi:10.1016/S1470-2045(17)30336-4

9. Holch JW, Demmer M, Lamersdorf C, et al. Pattern and Dynamics of Distant Metastases in Metastatic Colorectal Cancer. Visc Med 2017:33:70-75. doi:10.1159/000454687

10. Faron M, Pignon JP, Malka D, et al. Is primary tumour resection associated with survival improvement in patients with colorectal cancer and unresectable synchronous metastases? A pooled analysis of individual data from four randomised trials. Eur J Cancer 2015;51:166176. doi:10.1016/j.ejca.2014.10.023

11. Simillis C, Kalakouti E, Afxentiou T, et al. Primary Tumor Resection in Patients with Incurable Localized or Metastatic Colorectal Cancer: A Systematic Review and Meta-analysis. World J Surg 2019;43:1829-1840. doi:10.1007/s00268-019-04984-2

12. AJCC Cancer Staging Form Supplement.pdf. Accessed 2020 Jun 9 Available from: https://cancerstaging.org/references-tools/deskreferences/ Documents/AJCC\%20Cancer\%20Staging\%20Form\%20Supplement.pdf

13. Qiu M, Hu J, Yang D, Cosgrove DP, Xu R. Pattern of distant metastases in colorectal cancer: a SEER based study. Oncotarget 2015;6:38658-38666 doi:10.18632/oncotarget.6130

14. Mantke R, Schmidt U, Wolff S, Kube R, Lippert H. Incidence of synchronous liver metastases in patients with colorectal cancer in relationship to clinico-pathologic characteristics. Results of a German prospective multicentre observational study. Eur J Surg Oncol 2012;38:259-265. doi:10.1016/j.ejso.2011.12.013

15. Robinson JR, Newcomb PA, Hardikar S, Cohen SA, Phipps AI. Stage IV colorectal cancer primary site and patterns of distant metastasis. Cancer Epidemiol 2017;48:92-95. doi: 10.1016/j.canep.2017.04.003

16. Bara J, Nardelli J, Gadenne C, Prade M, Burtin P. Differences in the expression of mucus-associated antigens between proximal and distal human colon adenocarcinomas. Br J Cancer 1984;49:495-501. doi:10.1038/bjc.1984.77

17. Lee GH, Malietzis G, Askari A, Bernardo D, Al-Hassi HO, Clark SK. Is right-sided colon cancer different to left-sided colorectal cancer? - A systematic review. Eur J Surg Oncol 2015;41:300-308. doi:10.1016/j. ejso.2014.11.001

18. Weisenberger DJ, Siegmund KD, Campan M, et al. CpG island methylator phenotype underlies sporadic microsatellite instability and is tightly associated with BRAF mutation in colorectal cancer. Nat Genet 2006;38:787-793. doi:10.1038/ng1834

19. Yamauchi M, Morikawa T, Kuchiba A, et al. Assessment of colorectal cancer molecular features along bowel subsites challenges the conception of distinct dichotomy of proximal versus distal colorectum. Gut 2012;61:847-854. doi:10.1136/gutjnl-2011-300865

20. Benedix F, Kube R, Meyer F, et al. Comparison of 17,641 patients with right- and left-sided colon cancer: differences in epidemiology, perioperative course, histology, and survival. Dis Colon Rectum 2010;53:57-64. doi:10.1007/DCR.0b013e3181c703a4

21. Ducreux M, Malka D, Mendiboure J, et al. Sequential versus combination chemotherapy for the treatment of advanced colorectal cancer (FFCD 2000-05): an open-label, randomised, phase 3 trial. Lancet Oncol 2011;12:1032-1044. doi:10.1016/S1470-2045(11)70199-1

22. Jonker DJ, O'Callaghan CJ, Karapetis CS, et al. Cetuximab for the treatment of colorectal cancer. N Engl J Med 2007;357:2040-2048. doi:10.1056/NEJMoa071834

23. Seymour MT, Punt CJA. CAIRO and FOCUS. Lancet Lond Engl 2007:370:1904-1905. doi:10.1016/S0140-6736(07)61807-8

24. Tournigand C, Chibaudel B, Samson B, et al. Bevacizumab with or without erlotinib as maintenance therapy in patients with metastatic colorectal cancer (GERCOR DREAM; OPTIMOX3): a randomised, open-label, phase 3 trial. Lancet Oncol 2015;16:1493-1505. doi:10.1016/ S1470-2045(15)00216-8

25. Yoshino T, Mizunuma N, Yamazaki K, et al. TAS-102 monotherapy for pretreated metastatic colorectal cancer: a double-blind, randomised, placebo-controlled phase 2 trial. Lancet Oncol 2012;13:993-1001. doi:10.1016/S1470-2045(12)70345-5 
26. Hugen N, van de Velde CJH, de Wilt JHW, Nagtegaal ID. Metastatic pattern in colorectal cancer is strongly influenced by histological subtype. Ann Oncol 2014;25:651-657. doi:10.1093/annonc/mdt591

27. Segelman J, Granath F, Holm T, Machado M, Mahteme H, Martling A Incidence, prevalence and risk factors for peritoneal carcinomatosis from colorectal cancer. Br J Surg 2012;99:699-705. doi:10.1002/bjs.8679

28. van 't Sant I, Engbersen MP, Bhairosing PA, et al. Diagnostic performance of imaging for the detection of peritoneal metastases: a meta-analysis. Eur Radiol 2020;30:3101-3112. doi:10.1007/s00330-01906524-X

29. Hsu YL, Lin CC, Jiang JK, et al. Clinicopathological and molecular differences in colorectal cancer according to location. Int J Biol Markers 2019;34:47-53. doi:10.1177/1724600818807164

30. Ooi BS, Ho YH, Eu KW, Seow Choen F. Primary colorectal signet-ring cell carcinoma in Singapore. ANZ J Surg 2001;71:703-706. doi:10.1046/ j.1445-1433.2001.02269.x 